\title{
Hypothermia does Reduce Postoperative Adhesions: Reply
}

\author{
Cheng-Chung Fang $\cdot$ Tzung-Hsin Chou \\ Geng-Shiau Lin · Zui-Shen Yen • \\ Chien-Chang Lee $\cdot$ Shyr-Chyr Chen
}

Published online: 23 October 2010

(C) Société Internationale de Chirurgie 2010

\section{Sir,}

We thank Binda et al. for their interest in our study "Peritoneal infusion with cold saline decreased postoperative intra-abdominal adhesion formation" [1]. Our results do confirm the Binda et al. study that temperature change may affect adhesion formation in a laparoscopic mouse model [2]. Both studies showed that hypothermia reduces postoperative intra-abdominal adhesion formation.

Concerning the impact of cold saline infusion upon body temperature, we used continuous dripping of $4^{\circ} \mathrm{C}$ normal saline into the abdominal cavity until the cold saline ran out. The effect will be limited in the abdominal cavity; its effects on superficial layer or entire body are undetermined. We did not examine the changes of mesothelium; it would be valuable for another study to demonstrate the reaction of mesothelium. Our cytokine level is calculated as mean and standard deviation. Due to limited space, we did not provide those data. Blood sampling for cytokines in this study is at days 1, 3, 7, and 14. It is unclear whether days 7 or 6 have the highest level of cytokines. However, it is generally agreed that peritoneal repair is complete after 7 days. The levels of cytokines in group III is nearly the one to four times those of group IV; we are not sure it is only slightly changed of cytokines.

Finally, we do appreciate the excellent comments to clarify some intriguing issues and make the study of postoperative adhesion perfect.

\section{References}

1. Fang CC, Chou TH, Lin GS et al (2010) Peritoneal infusion with cold saline decreased postoperative intra-abdominal adhesion formation. World J Surg 34(4):721-727

2. Binda MM, Molinas CR, Mailova K et al (2004) Effect of temperature upon adhesion formation in a laparoscopic mouse model. Hum Reprod 19(11):2626-2632
C.-C. Fang · T.-H. Chou · G.-S. Lin · Z.-S. Yen · C.-C. Lee ·

S.-C. Chen $(\bowtie)$

Department of Emergency Medicine, National Taiwan

University Hospital and National Taiwan University

College of Medicine, Taipei, Taiwan

e-mail: scchen@ntu.edu.tw 